\title{
STUDENT MENTAL WELLBEING INTERVENTIONS WITH A SECOND-YEAR ENGINEERING COHORT
}

\author{
Agnes G. d'Entremont ${ }^{1}$, Jocelyn Micallef $f^{2}$, Gabriel Smith ${ }^{2}$, Juan Abellól ${ }^{1}$, and Diana Jung ${ }^{2}$ \\ ${ }^{1}$ Department of Mechanical Engineering, ${ }^{2}$ Health Promotion and Education, University of British Columbia \\ adentremont@mech.ubc.ca, abello@mech.ubc.ca, diana.jung@ubc.ca
}

\begin{abstract}
Concern about student mental wellbeing in university students is high, and first- and second-year students in particular have shown higher levels of depression, anxiety and stress. Evidence indicates that there is value in context specific mental health literacy interventions embedded into regular academic classes, and that coping strategies help students to not only deal with stress but also to improve cognitive motivation and academic achievement.

Within our second-year mechanical engineering academic program, we implemented an embedded series of interventions with the goal of helping students develop and use coping strategies in the face of stress.

We found that students could describe their own stress response and coping strategies, and over $80 \%$ expected content on stress response and coping strategies would be useful in their own lives. About half of students viewed the interventions as beneficial to them. A third of students expected the activities would positively impact their academics, while another third felt they would have no impact.

Test anxiety increased over the year, which we felt was a reasonable result given the rigour of the program. Since we did not have a control group, we could not determine whether our intervention led to lower levels of test anxiety than would have otherwise occurred. Our results suggest that academic buoyancy (day-to-day resilience in an academic program) is a critical skill - low academic buoyancy was a significant predictor of increased test anxiety, while low self-efficacy was not. This finding, along with student responses indicating the usefulness of the interventions, support our approach of introducing coping skills into an academic engineering program.
\end{abstract}

Keywords: Mental health, stress, intervention, mental wellbeing, workshop, stress response, coping strategies, mental health literacy.

\section{INTRODUCTION}

Students attending Canadian engineering programs are often accustomed to achieving high marks, as the competitive application process in many schools leads to relatively high incoming grades. Similarly, schools with common first-years and competitive entry to disciplines can see similar cohorts composed primarily of highachieving students in popular second-year programs. As students reach these more competitive programs, half of them now find they are scoring below the class average and earning marks below $80 \%$ for the first time. Anecdotally, engineering instructors observe a great deal of student stress in these cohorts.

As we detailed in a previous review of the literature [1], there has been a marked increase in awareness around mental health concerns and counselling usage at Canadian universities [2]. Many studies indicate both high levels of stress-related mental health issues, and low rates of treatment [3]. For example, in 2011, Queen's University found that $60 \%$ of students reported above average or "tremendous" stress levels, and $62 \%$ of all students reported that consequences of this stress included mental health problems. The largest reported source of stress was academic pressures (73\%) [4]. First- and second-year students in particular have been shown to have higher depression, anxiety and stress scores than other university students [5].

Literature has shown that interventions designed to increase mental health literacy in post-secondary students - including understanding how to develop and maintain positive mental health - can have a positive impact on helpseeking efficacy and stress reduction in student populations [3], [6].

In recent research, post-secondary students at a Canadian institution reported negative attitudes towards help-seeking, but had interest in learning about symptoms and coping strategies [7]. Additional Canadian research has shown that mental health literacy programming is context dependent [8] and that there is value in embedding content in regular classes, delivered by the regular teacher [9]. A study of engineering students found that coping strategies are key for not only students' ability to deal with stress but also for cognitive motivation and academic achievement [10].

As part of a larger, campus-wide initiative exploring student wellbeing in academic environments, our secondyear mechanical engineering program (124 students) partnered with the university health promotion and education (HPE) unit to develop and deploy three interventions in the fall term of 2018. The delivery of these interventions was led by an engineering instructor (author $\mathrm{AD})$. They were embedded in the regular second-year 
academic program, and were focused on coping strategies in the face of stress.

In this paper, we will present details on the interventions and an analysis of student responses to a reflection exercise and survey results from students.

\section{METHODS}

Three interventions of varying scale were deployed over the term. The first intervention was a short information session about resources on campus, given at the start of the term. The second intervention was a reflection exercise based on a video about stress and the stress response, deployed in late September. And the final intervention was a workshop on stress and methods for reducing the acute stress response in order to allow students to then respond to the underlying stressor, deployed in late October.

Data collection included four surveys, and an analysis of submitted reflection responses. Two surveys (late September and late March) were pre- and post-intervention measures comprised of several scales used to assess specific social and psychological constructs. These surveys were common to all HPE pilot projects at the university. One survey after the workshop focused on the workshop only, and another survey at the end of the term asked about feedback on all the interventions.

\subsection{Information session}

On the first day of classes for the program, a 15-minute overview of general health and wellness programs on campus was presented by an HPE specialist. This was a new addition to the regular half-day welcome and orientation. Due to a substantial transfer cohort entering $\mathrm{UBC}$ at the second-year level through the BC Engineering Transfer Program and other from other campuses $(22.4 \%$ reported completing their first-year studies elsewhere), many students would not be familiar with the resources on campus. This orientation would have been the first (and possibly only) formal introduction of these transfer students to the campus health and wellness milieu.

The primary themes of the information session were 1) be proactive by building and maintaining your health so that you are better prepared for challenges, 2) talk to someone when you are feeling overwhelmed, and 3) help a peer or a friend if you notice them struggling. A holistic approach to wellness was described to include all areas of life (including social, spiritual, academic, financial, physical, etc.).

In-person and online campus resources and services were highlighted, including websites, peer support programs, advisors, medical personnel, and counselling services.

\subsection{Initial survey}

The initial survey, deployed in late September, was comprised of several scales and sub-scales measuring factors that may be related to mental wellness in academic settings. The content of this survey was determined as part of the set of campus-wide pilot projects being coordinated by the HPE unit.

The Motivated Strategies for Learning Questionnaire (MSLQ) [11] provided subscales on:

1. Self-efficacy for Learning and Performance (MSLQ-SLP)

2. Intrinsic Goal Orientation (MSLQ-IGO)

3. Extrinsic Goal Orientation (MSLQ-EGO)

4. Test Anxiety (MSLQ-TA)

The university's Undergraduate Experience Survey subscales on Belonging (UES-B) and self-reported Mental Health (UES-MH) were also included (Appendix A:). Finally, a scale on Academic Buoyancy (AB), or day-today resilience in an academic setting (as opposed to resilience in the face of major events), was included [12].

\subsection{Video and reflection}

In the second intervention, students were asked to view a 15-minute TED talk by Kelly McGonigal on the effects of reframing the stress response [13]. In this TED talk, McGonigal refers to a 2012 study showing that high stress correlates to an increased risk of dying, but only for people who believe stress is harmful [14]. McGonigal also cites a 2013 study showing that thinking about the physical stress response (e.g. faster breathing and heartbeat) as helpful (the body is preparing to rise to a challenge) prevents the harmful effects (blood vessel constriction) that would otherwise accompany a stress response [15]. This activity took place in late September/early October.

Watching this TED talk was a voluntary, unmarked assignment for students to complete outside of class time. Students who watched the video then completed a reflection exercise, where they provided 0.5-2 pages of written answers to open-ended questions (Appendix B:; students were told that completion would be considered in grading decisions if their final mark was on a grade boundary). These questions were intended to help students reflect on the ideas presented in the McGonigal TED talk (how students personally respond to stress, how they could reframe this response as their body preparing to overcome a challenge) and recall some key wellness ideas presented in the first-day information session (where to go for support, how to help their peers.)

\subsection{Reflection analysis}

In an effort to further our knowledge of student experiences with stress, their understanding of stress, and their coping strategies, one author (JM) performed a qualitative analysis of the student reflection responses submitted. 


\subsection{Stress response workshop}

As part of our second-year program, we have 4 or 5 onehour slots every year where we bring in guest speakers and run workshops (for example, we have had past workshops on equity and on LaTeX). We set aside one of these speaker slots in mid-October for a workshop on the physical stress response and coping strategies. The workshop was cofacilitated by a subject-matter expert from HPE (author DJ) and an engineering instructor (author AD).

The workshop started with a sharing activity, where students traded five sticky notes written with something they personally do to recharge/refocus/regain balance. We had them brainstorm the physical and cognitive impacts of stress, then the subject-matter expert presented on the stress response and three evidence-based coping strategies: managing the intensity of the stress response, reframing, and problem-solving. Students were given handouts on creating action plans related to the coping strategies.

\subsection{Stress response workshop survey}

At the end of the workshop, we asked students to complete an online survey in the classroom. This survey contained one open-ended and several Likert-scale questions. Students rated the novelty and relevance of the workshop content to their personal and academic life, and reflected how (and whether) they could incorporate the coping strategies from the workshop into their academic endeavour. One author (AD) performed quantitative analysis of Likert-scale responses and qualitative analysis of open responses to this survey.

\subsection{Survey on past and potential interventions}

The purpose of this survey, delivered in November/December was to gather specific feedback on the collection of interventions deployed and determine their perceived utility and relevance for students, and what students thought we should do in the future. This survey contained both Likert-scale and open-ended questions.

One author (JA) performed a quantitative analysis on the Likert-scale questions, and qualitative analysis of open responses to this survey.

\subsection{Final survey}

The final survey (March 2019), included the same items as the initial survey, with the intention to measure pre- and post-intervention results. One author (GS) performed quantitative analysis on the pre- and post-survey data.

\section{RESULTS}

\subsection{Initial and final survey}

Without a control group it was impossible to directly test the effectiveness of the program in terms of key outcome measures, but responses on the surveys at the beginning and end of the semester were compared in order to examine trends in student wellbeing over the course of the semester. While student numbers were used to link survey responses across time, only 16 students answered the survey at both times, rendering paired analysis unreliable. As such, we made the choice to discard the pretest scores of these 16 students and treat the pre-test responses as being independent from the post-test responses, thus conducting independent as opposed to dependent-sample t-tests to determine whether the two groups were different.

Average test anxiety was the only statistically different measure $(\alpha=0.05)$ between the pre (September 2018) and post (March 2019) deployments of the survey. Average test anxiety increased dramatically from a mean rating of 3.77 at the beginning of the year to a mean of 4.64 (out of 7) at the end $(\mathrm{t}(56)=-2.26, \mathrm{p}=0.027)$. Belonging scores showed a nearly-significant decrease $(\mathrm{t}(55)=1.98, \mathrm{p}=$ 0.052 ) from 4.52 at the beginning of the year to 4.12 (out of 6 ) at the end.

In order to guide the stress management elements of the intervention in the future, a post-hoc analysis to determine whether certain aspects of anxiety were more responsible for the observed effects was conducted. The five questions composing the anxiety scale were broken up into the three that index the cognitive component of anxiety (i.e., anxious thought patterns) and the two that relate to the somatic/affective components (i.e., physical feelings and general emotions), and the same independent-sample t-test was conducted on each component. This analysis showed that only the cognitive component showed a significant increase over time $(\mathrm{t}(56)=-2.50, \mathrm{p}=0.015)$. (We should also note that there was a non-significant trend towards the somatic/affective component increasing over time $(\mathrm{t}(55)=$ -1.38, $\mathrm{p}=0.172$ ).

An exploratory correlation analysis was conducted to examine the relationships between the variables of interest (Table 1). (As in the analysis above, pre-test scores for those who responded at both times were removed to maintain independence).

In order to examine the variables that showed changes across the semester, two regression analyses were conducted. Test anxiety and belonging were the outcome, and the variables associated with each were the predictors.

For test anxiety, the only variable that maintained significant predictive power once all others had been accounted for was academic buoyancy; belonging, mental health, and self-efficacy all become non-significant in the multiple regression. These results appear in Table 2, where $\beta$ is the standardized regression coefficient. 
Table 1: Correlation analysis

\begin{tabular}{|l|c|c|c|c|c|c|c|}
\hline & Grade & $\begin{array}{c}\text { Academic } \\
\text { Buoyancy }\end{array}$ & Self-Efficacy & Test Anxiety & $\begin{array}{c}\text { Intrinsic } \\
\text { Motivation }\end{array}$ & $\begin{array}{c}\text { Extrinsic } \\
\text { Motivation }\end{array}$ & Belonging \\
\hline Grade & & & & & & & \\
\hline $\begin{array}{l}\text { Academic } \\
\text { Buoyancy }\end{array}$ & $0.42^{*}$ & & & & & & \\
\hline Self-Efficacy & 0.17 & $0.39^{*}$ & & & & & \\
\hline Test Anxiety & -0.23 & $-0.56^{*}$ & $-0.35^{*}$ & & & & \\
\hline $\begin{array}{l}\text { Intrinsic } \\
\text { Motivation }\end{array}$ & 0.25 & 0.12 & $0.50^{*}$ & 0.04 & & \\
\hline $\begin{array}{l}\text { Extrinsic } \\
\text { Motivation }\end{array}$ & -0.04 & -0.12 & -0.07 & 0.20 & 0.00 & & \\
\hline Belonging & 0.29 & $0.59^{*}$ & $0.25^{*}$ & $-0.37^{*}$ & $0.29^{*}$ & -0.14 & -0.07 \\
\hline $\begin{array}{l}\text { Mental } \\
\text { Health }\end{array}$ & $0.47^{*}$ & $0.49^{*}$ & $0.26^{*}$ & $-0.32^{*}$ & $0.35^{*}$ & $0.61^{*}$ \\
\hline
\end{tabular}

* indicates predictors that are significantly associated with the outcome after accounting for other variables.

Table 2: Multiple regression results predicting test anxiety.

\begin{tabular}{|l|c|c|}
\hline Predictor & $\boldsymbol{\beta}$ & $\mathbf{p}$ \\
\hline Academic Buoyancy* & -0.47 & 0.002 \\
\hline Belonging & -0.03 & 0.829 \\
\hline Mental Health & -0.03 & 0.853 \\
\hline Self-Efficacy & -0.13 & 0.308 \\
\hline
\end{tabular}

* indicates predictors that are significantly associated with the outcome after accounting for other variables.

Similarly, academic buoyancy and mental health scores both independently and significantly predicted belonging, while self-efficacy, intrinsic motivation, and test anxiety become non-significant predictors. These results appear in Table 3 , where $\beta$ is the standardized regression coefficient.

Table 3: Multiple regression results predicting belonging.

\begin{tabular}{|l|c|c|}
\hline Predictor & $\boldsymbol{\beta}$ & $\mathbf{P}$ \\
\hline Academic Buoyancy* & 0.39 & 0.005 \\
\hline Intrinsic Motivation & 0.18 & 0.183 \\
\hline Mental Health* & 0.36 & 0.005 \\
\hline Self-Efficacy & -0.12 & 0.380 \\
\hline Test Anxiety & -0.07 & 0.585 \\
\hline
\end{tabular}

* indicates predictors that are significantly associated with the outcome after accounting for other variables

\subsection{Reflection analysis}

Forty-nine students completed the assignment between September $26^{\text {th }}$-October $6^{\text {th }}$. The responses to these 6 sections were organized into three broad themes: (1) How do students understand stress? (2) How do students perceive the relationship between stress and life? (3) How do students cope with stress? (including What do students know about resources?). A high-level summary of each theme, and of student reactions to the activity, is included below.

3.2.1. How do students understand stress? When prompted, students shared what stress felt like in their own bodies. While a small subset of students did not personalize their responses, and instead spoke to how stress might feel more generally, most students appeared to have a nuanced understanding of how stress manifests in themselves.

Students' responses captured a variety of both physical and mental signs of stress including changes to appetite, body temperature, heart rate, breathing, sweating, immunity, sleep, headaches. Some students provided a complex description of stress, and differentiated experiences with different kinds of stress.

3.2.2. How do students perceive the relationship between stress and life? Many students drew connections between feelings of stress and their lives, including their academics. Activities such as interviews, test-taking and presentations occurred in many of their responses related to stress. Some students acknowledged stress explicitly as normal. Further, students linked stress to both positive and negative outcomes. Some negative connections that students mentioned included fear, anxiety and negative academic outcomes. However, one student conceived as stress as a reminder to remove stressors, and suggested the presence of stress could improve focus. Another student shared how mindset, and appraisal of the situations mattered for the outcome of stress.

3.2.3. How do students cope with stress? Students shared a variety of ways that they currently cope with stress including: rest, physical activity, reading, relaxing activities, apps, music, problem solving, religion, volunteering and social support. Social support was a prominent strategy shared. Students indicated that they would draw on friends and family for social support, some students indicated that they would go to their peers for support, and faculty or TA for academic help if the stressor was related. Students differentiated between who they would go to for different kinds of support. For example, "TA's and professors can provide academic help, while councillors can provide emotional help."

Many students shared similar strategies for supporting others, including providing social support, problem 
solving, sharing what they learned from this assignment about the purpose of the stress response, or connecting to a professional. Considering reaching out to friends and families was such a frequently indicated strategy, some students also drew attention to challenges in relying on networks of support, and referred to their own experience of having friends as luck.

When students shared their strategies for coping with stress, they often referred to professional help, and 23 of the 49 students referred to resources on campus (e.g., counselling services, faculty and TAs, the Wellness Centre, EmpowerMe, Student Services, Engineering Student Services, AMS Speakeasy).

3.2.4. Students' reaction to the activity. Many students were successful in reframing the stress response in a positive way, that aligned with the video content. Some students demonstrated that they were grappling with thinking about how applying the content can be challenging. When asked to explain the stress response in his/her own words to a friend who was struggling with stress, many students drew directly on the content from the video, some even reporting that they would share the video. In these responses, stress was described in many ways, including as a warm-up, as a notification of a stressor and as normal.

\subsection{Stress response workshop survey}

Ninety-nine students completed this survey. The simple selection or Likert-scale questions indicated close to $60 \%$ (57/99) of respondents watched the Kelly McGonigal TED talk (section 2.3) prior to the workshop. Close to $40 \%$ (37/99) of respondents indicated the content presented at the workshop was new to them, and over $80 \%$ indicated this content was useful to their personal (85/99) and academic (82/99) lives.

Sixty-six of these 99 students provided answers to the open-ended question asking them to what extent the content of the workshop was applicable to their academic work. A qualitative analysis of the answers follows.

About a third of respondents (23/66) appreciated the workshop because it taught them coping strategies, with about $10 \%$ of respondents (7/66) indicating these techniques were important to prevent health issues or academic challenges in the future. In contrast, a little over $10 \%$ of respondents $(12 / 66)$ were unsure of the usefulness of the workshop or did not find the workshop useful.

About one fifth of respondents (12/66) felt the coping strategies in the workshop were useful in their academic life, while a handful (4/66) said they were also useful in their personal life. A couple of students said they were already familiar with the content of the workshop, but one of them appreciated hearing about it from a professional psychologist.

Interestingly, about $20 \%$ of respondents (14/66) view stress as an unavoidable and endemic part of academics.

\subsection{Survey on past and potential interventions}

Twenty students answered this survey, although not all of them provided answers to all open-ended questions. A qualitative analysis of the open-ended student responses indicates is presented below.

About one third of respondents (6/17) enjoyed watching the Kelly McGonigal TED talk (section 2.3) and about one third (6/17) enjoyed the guest speaker in the stress response workshop (section 2.5). Interestingly there was little overlap (1/17) between these two groups.

About half of respondents (7/16) see learning about the stress response as useful because it helps them better understand how to cope with stress, and about one third (5/16) see it as helpful for improving academic performance. Another (approximate) third (5/16) believe that learning about wellness and stress management has no effect on their academic work.

Almost half of respondents (8/18) felt the wellness interventions incorporated into the course had a positive effect on their student experience, with about half of these students (4/8) indicating the interventions had provided tools to deal with stress. The other (almost) half of respondents $(8 / 18)$ felt the interventions were not helpful to their experience.

When asked for suggestions on how to improve the wellness interventions, almost a third of students (4/13) requested bonus marks be assigned for participating in these activities. This was the most popular change request from students.

When asked what other activities students would like to see in the future, half of respondents (4/8) leaned towards more wellness activities while the (almost) other half (3/8) preferred fewer or none. Among students who wished for more activities, half (2/4) suggested more practical exercises while the other half (2/4) preferred more guest lectures on how to deal with stress.

The Likert-scale questions provided results that were consistent with the qualitative analysis shown above.

\section{DISCUSSION AND CONCLUSIONS}

We developed, deployed, and assessed a series of mental wellness activities embedded in a second-year mechanical engineering academic program. Students showed a nuanced understanding of their own stress response, and could identify coping strategies. The majority of students indicated that the workshop content would be useful in both their personal and academic lives. Anecdotally, faculty and staff involved in program coordination and advising saw a decrease in the number of visits from students in emotional distress due to academics than they had in the past two years, but this was not quantified, nor could it be linked directly to the interventions.

This pilot project has indicated that there is likely value in embedding mental health literacy, and specifically 
coping skills development, into the engineering academic curriculum. We framed stress as something students must learn to manage, both in school and in their future careers as engineering professionals, and provided opportunities for evidence-based knowledge and skills development.

Across the pre-post surveys we found an increase in test anxiety. The increase in test anxiety is not unexpected given the intense performance pressure placed upon students entering our integrated second-year mechanical engineering program. We believe that both the qualitative findings and the proportion of students who reported the content of the workshops was useful is good preliminary evidence that the intervention helped to dampen the effects that entering the program has on wellbeing.

Further, when breaking down the questions of the test anxiety scale into those that reflect the cognitive component of anxiety from the somatic/affective components, we found that only the cognitive component showed a significant increase over time. Stress-reframing techniques such as those used in the intervention were designed to target the cognitive component of anxiety. This suggests that the approach we took in using these techniques may correctly target a primary area of concern.

The interventions in this program were developed emergently, in response to needs perceived by engineering instructors. As we look forward to a new iteration of the program, we have a number of reflections on the strengths and challenges of this approach.

The interventions were pursued through a collaborative partnership between regular instructors and HPE specialists. Aligned with the literature [9], we recognized the strength of delivering content primarily through the regular course curriculum, by the course instructor (author $\mathrm{AD})$. By embedding the content into the curriculum, the instructor - an expert and professional in the field - models the importance of considering stress and coping strategies as part of being an engineer. This regularizing of mental health content may also decrease the stigma of talking about stress and related issues. Where this author (AD) took the lead on content delivery, a content-expert (author DJ) co-facilitated key workshops components. The collaboration in developing and delivering the interventions allowed for health promotion expertise to be operationalized in a mechanical engineering context, where examples and content were designed flexibly to be relevant to the audience.

There is a clear opportunity to refine our understanding on the impacts of our interventions, through the update of evaluation techniques. For instance, due to the emergent design of the program, we did not have a control group, which limited the scope of available analyses. However, we may be able to compare some aspects of the intervention cohort through answers to the UES questions, which are administered to all students (including all other second-year engineering students) at our institution. Further, there was low uptake on each of the surveys, resulting in an inability to trace a student's journey across the program. Completing evaluations during class time and incentivizing these evaluations appropriately may improve uptake.

In terms of students' skill development, we did not ask for students to report help-seeking behaviours (i.e., the number of times they reached out to a peer or campus resource before or throughout the time of these interventions). This information could help inform the efficacy and experiences of students' uptake of resources.

With regards to students' sense of belonging, we included survey questions that provided us insight into students' connection on campus, however adding questions related to the program itself would be necessary for understanding students' sense of connection to their program and peers. Student formal and informal feedback (including testimonials [16]) explicitly mention the strong community development within the second-year mechanical engineering program. It is reasonable that sense of belonging on the institution level may diminish as sense of belonging on the program or cohort level may increase. Relatedly, we know that $22 \%$ of the students in this program transferred into the engineering program at the Vancouver campus, however we did not ask students to self-report this detail in relation to their survey responses. Including this detail would have supported us in understanding the impact of the interventions in relation to this specific group, particularly in relation to sense of belonging.

Given these responses, and the portion of students who reported the intervention as relevant to both their academic and personal lives, there is an opportunity to explore the effects of the interventions more fully. Further exploration of how students develop skills (including strategies for coping), cultivate belonging (including relationships in the classroom), and develop both motivation for learning and mindset around professional aspects of being an engineer may be beneficial.

\section{Acknowledgements}

We gratefully acknowledge the financial support for this project provided by UBC Vancouver students via the Teaching and Learning Enhancement Fund.

We would like to thank the 2018-2019 Mech 2 students who participated in the activities and evaluations during this project. We would also like to thank Patty Hambler (Health Promotion \& Education), Natasha Moore (UBC Wellbeing), and other members of their units for supporting this project.

This work was completed on the traditional, ancestral, and unceded territory of the Musqueam people. 


\section{References}

[1] A. G. d'Entremont and J. Abelló, "Being a C student is the new normal: a literature review on grades, selfworth, and mental wellbeing," in CEEA-ACEG Annual Conference, 2018, p. Paper 133.

[2] A. Hutchins, "Are universities doing enough to support mental health? - Macleans.ca," MacLean's, 2017. [Online]. Available: http://www.macleans.ca/education/depth-of-despair/.

[3] C. Regehr, D. Glancy, and A. Pitts, "Interventions to reduce stress in university students: A review and metaanalysis,” J. Affect. Disord., vol. 148, pp. 1-11, 2013.

[4] Queen's University, "Student Mental Health and Wellness Report of the Principal's Commission on Mental Health," pp. 1-12, 2012.

[5] N. Bayram and N. Bilgel, "The prevalence and sociodemographic correlations of depression, anxiety and stress among a group of university students," Soc. Psychiatry Psychiatr. Epidemiol., vol. 43, no. 8, pp. 667-672, 2008.

[6] N. Reavley and A. F. Jorm, "Prevention and early intervention to improve mental health in higher education students: a review," Early Interv. Psychiatry, vol. 4, pp. 132-142, 2010.

[7] L. L. Armstrong and K. Young, "Mind the gap: Personcentred delivery of mental health information to postsecondary students," Psychosoc. Interv., vol. 24, no. 2, pp. 83-87, 2015.

[8] S. Kutcher, Y. Wei, and C. Coniglio, "Mental Health Literacy: Past, Present , and Future," Can. J. Psychiatry, vol. 61, no. 3, pp. 154-158, 2016.

[9] S. Kutcher, Y. Wei, and C. Morgan, "Successful application of a Canadian mental health curriculum resource by usual classroom teachers in significantly and sustainably improving student mental health literacy," Can. J. Psychiatry, vol. 60, no. 12, pp. 580-586, 2015.

[10] P. H. Hsieh, J. R. Sullivan, D. A. Sass, and N. S. Guerra, "Undergraduate engineering students beliefs, coping strategies, and academic performance: An evaluation of theoretical models," J. Exp. Educ., vol. 80, no. 2, pp. 196-218, 2012.

[11] P. Pintrich, D. Smith, T. García, and W. McKeachie, "Manual for use of the motivated strategies for learning questionnaire," pp. 1-75, 2002.

[12] A. J. Martin and H. W. Marsh, "Academic buoyancy: Towards an understanding of students' everyday academic resilience," J. Sch. Psychol., vol. 46, no. 1, pp. 53-83, 2008.

[13] K. McGonigal, "How to make stress your friend," 2013. [Online].

Available: https://www.ted.com/talks/kelly_mcgonigal_how_to_m ake_stress_your_friend.

[14] A. Keller, K. Litzelman, L. E. Wisk, T. Maddox, E. R. Cheng, P. D. Creswell, and W. P. Witt, "Does the perception that stress affects health matter? The association with health and mortality.," Heal. Psychol., vol. 31, no. 5, pp. 677-684, 2012.

[15] A. J. Crum, P. Salovey, and S. Achor, "Rethinking stress: The role of mindsets in determining the stress response.," J. Pers. Soc. Psychol., vol. 104, no. 4, pp. 716-733, 2013.

[16] “Community, UBC Mechanical Engineering." [Online]. Available:

https://mech.ubc.ca/undergraduate/community/.

\section{APPENDIX A: UES SURVEY ITEMS}

The Undergraduate Experience Survey is a biannual, institution-wide survey. Two subscales from this survey were used in the pre- and post-surveys in this study. Participants could rate each item on a 6-point Likert scale between "strongly agree" and "strongly disagree". Note that the Belonging subscale asks only about belonging at the university level, and not the program level, and the Mental Health and Wellbeing also asks only about care at the university level.

\section{A.1 UES Belonging}

Please rate your level of agreement with the following statements:

- I feel a strong sense of connection to UBC

- I feel I belong at UBC

- I am confident that I have the ability to succeed in all of my courses

- I am able to balance my academic time (in class, study time, etc.) and non-academic time (work, exercise, socializing, care for dependents, etc.)

- UBC provides a supportive learning environment

- UBC uses teaching strategies designed to support learners

- I feel that I belong on campus

- I feel part of a campus community

\section{A.2 UES Mental Health and Wellbeing}

Please rate your level of agreement with the following statements:

- I'm confident in my ability to cope with the demands of my life

- Since starting at UBC, I've gained new skills to help me manage the demands of university life

- I'm aware of personal signs when I experience too much stress

- I know about strategies I can use to cope with stress as it comes

- This school year, I've been able to manage stress successfully

- I'm comfortable reaching out for support when I need it

- UBC cares about my wellbeing 


\section{APPENDIX B: VIDEO REFLECTION}

This video activity was intended to (a) help students understand what the stress-response is (first three prompts), and (b) help students understand what purpose the stress response has (last three prompts).

\section{B.1 Video reflection prompt and questions}

In Kelly McGonigal's TedTalk she speaks to both the physical stress response, and individual's beliefs about the stress response. She explains that our beliefs about the stress response have an impact on how stress is manifested in the body. She explains that normal physical responses to stress include a quickened heartbeat - however, only when we believe that stress is bad for us, do blood vessels constrict (potentially leading to coronary issues).

1. Take note of how your physically feel when you are experiencing stress - What changes do you notice in your body?
In her talk, she mentions that it is common to associate these changes with negative signs of coping, however, she these signs could be viewed differently, as a sign that there is a challenge that you are prepared to address.

2. What do you currently associate with each of these responses?

3. How could you think of these responses as your body preparing to be courageous in the face of a challenge?

Kelly McGonigal refers to the stress hormone, oxytocin, as key in promoting social support in times of stress.

4. In times of stress, where can you go to receive support?

5. When you notice that others are stressed, how can you support them?

6. In your own words, how would you explain the stress response, and the purpose of the stress response to a friend who was struggling with stress? 